\title{
Sharing spaces, sharing lives - the impact of robot mobility on user perception of a home companion robot
}

\author{
Dag Sverre Syrdal, Kerstin Dautenhahn, Kheng Lee Koay, Michael L. Walters and \\ Wan Ching Ho \\ Adaptive Systems Research Group, School of Computer Science, University of Hertfordshire, \\ Hatfield, UK
\{d.s.syrdal;k.dautenhahn;k.l.koay;m.l.walters\} @herts.ac.uk, wanchingho@gmail.com

\begin{abstract}
This paper examines the role of spatial behaviours in building human-robot relationships. A group of 8 participants, involved in a long-term HRI study, interacted with an artificial agent using different embodiments over a period of one and a half months. The robot embodiments had similar interactional and expressive capabilities, but only one embodiment was capable of moving. Participants reported feeling closer to the robot embodiment capable of physical movement and rated it as more likable. Results suggest that while expressive and communicative abilities may be important in terms of building affinity and rapport with human interactants, the importance of physical interactions when negotiating shared physical space in real time should not be underestimated.
\end{abstract}

\section{Motivation}

The study presented in this paper is part of the on-going work being done in the University of Hertfordshire (UH) Robot House to perform early prototyping of longterm Human-Robot Interaction (HRI) in domestic environments. There is a growing interest in the use of robots as assistive companions in domestic environments [1], however, there are many challenges that need to be overcome in order for robots to be not only useful in these settings, but also acceptable to their users. While all adoption of novel technologies in domestic settings may be disruptive, autonomous robots may be especially so, in particular if they are mobile [2]. The ability to move autonomously makes them qualitatively different from other household appliances. This ability allows for a wider range in functionalities, such as the ability to assist their use when moving [3], and transporting objects [4,5]. It may also confer advantages when obtaining information from its environment when compared to static technologies, which may make them more suitable for safe-guarding the health of their users [6,2].

However, having technological artefacts that move in a shared space with human residents is not without its problems. Hüttenrauch and Severinson Eklundh's [4] study highlighted instances where negotiation of space caused annoyance and discomfort in 
a professional environment, and movement in a human-centred environment have inherent safety concerns [7]. In addition, having to share spaces with robots in domestic environments may cause discomfort if the robot does not conform to social norms for proxemics behaviour [8]. This may be of particular concern if the robot is perceived to be a social actor [9].

\subsection{Socially Assistive Still Robots}

There are several robots with functionalities that do not rely on the ability to navigate around the environment of its user. In terms of market-ready products, the zoomorphic PARO has been shown have an impact on the emotional well-being of its users. Studies using the similarly zoomorphic Philips iCat [10] found that it could be used to engage elderly people in a care home for in conversation. The Autom weight loss coach produced by Intuitive Automata offers advice and attempts to persuade its users in making decisions that are conducive to a healthier life style [11].

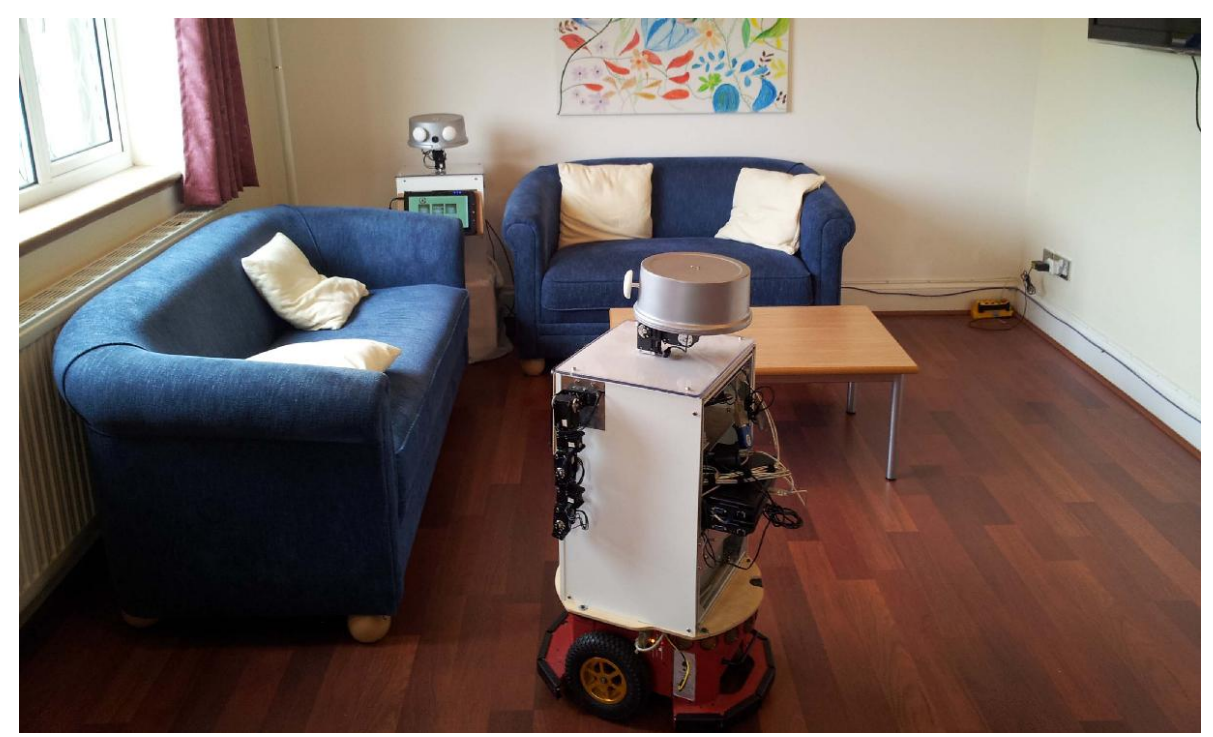

Fig. 1. Robot House Living Room Area. It shows one mobile Sunflower robot in the front, and a stationary Sunflower robot in the back.

\subsection{The importance of being mobile}

There are however, important benefits for a social robot in being able to move in a shared space with human interactants. If one was to consider human-robot relationships in terms of their anthropomorphic counterparts, one could most certainly make this case. In human-human interactions, proxemic behavior and interpersonal spacing is a highly communicative act [12]. Kendon [13] gives several examples of how humans manage and signal the quality and nature of their interactions through continuous maintenance of appropriate spatial behaviour. Even when we engage in it 
Sharing spaces, sharing lives - the impact of robot mobility on user perception of a home companion robot 3

in an unconscious manner, we continuously validate and define the nature of our relationship with those we interact with. Hall [14] and Mehrabian [15] both offer evidence of proxemic behaviour as indicative of the interactants' relationship, mutual attitude and relative status to each other. In fact, Burgoon and Walther [16] suggest that proxemics behavior can dramatically alter the nature of our relationships, and that changes in how we feel or reason about the people we interact with depend on responses to such changes in proxemic behaviour to take effect.

With such richness in human-human interaction being dependent on this spatial interactional dimension, it seems that even for robots that may not strictly be required to navigate autonomously in order to perform their functions, this ability may be of benefit from a purely interactional point of view.

Stienstra and Marti [17], when considering the possibility of emergent humanmachine empathy, highlight the importance of going beyond the explicitly symbolic interactions that are most commonly associated with human-machine interactions and instead focus on interaction modalities that allow for a rich and continuous loop of mutual action and perception. Instead of sharing one's intentionality through constrained voice commands or navigating menus, moving within the same space as the robot is a continuous interaction that allows for synchronization between human and robot interactant in an authentic manner, based on actual behavioral affordances for both parties, through a series of small-scale epistemic actions [18] that occur in addition to the large-scale tasks. In this perspective, the ability to negotiate shared spaces allows for experiences that are seen as shared, both in terms of perception as well as behaviour, which in turn allows for greater feelings of mutual understanding and empathy in the human interactant.

The above argument is supported by HRI research regarding the role of physical embodiment. Wainer et al. [19] found that both task performance and social perceptions of a robot benefited from interacting in shared physical space. KoseBagci et al. [20] found a similar effect using a humanoid robot in a synchronization task. In socially assistive robotics, Tapus et al. [21], provided some evidence that changes in proxemic behaviour was an effective way to allow for personalization in stroke rehabilitation therapy.

This suggests that an examination into the role of the ability to move and share the physical space with the user in the formation of human-robot relationship is a valid avenue of investigation, in particular when comparing to robotic embodiments that have the same physical expressive capabilities, with the exception of gross physical movement, which is only possessed by one robot embodiment.

\section{Method}

\subsection{Participants}



Wan Ching Ho

Eight participants were recruited via advertisements on the University Intranet, 3 males and 5 females. The mean age was 25 , the oldest participant was 32 and the youngest was 21 .

\subsection{Apparatus}

UH Robot House. The UH Robot house was built as a residential house in a neighbourhood near the University of Hertfordshire campus, which has been adapted for Human-Robot Interaction studies, including low-cost, resource-efficient sensor systems to inform robots about user-activities and other events in the environment [22]. In the course of the current study, participants would spend time in the Kitchen, Dining, and Living Room areas of the house.

Sunflower Robots. This study used two UH Sunflower robots. The UH Sunflower is built on top of a commercially available Pioneer P3-DX mobile base. Its custom-built superstructure includes as an expressive head with a static face, a speaker capable of playing midi tunes and a diffuse color LED display panel. In addition it has a slide-out carrying tray, which can be used for transporting objects, and an integrated touch screen user interface for menu-driven interaction displaying messages. Apart from gross body movements such moving back and forwards and moving its base from side to side, the UH Sunflower has several expressive channels for expressing its internal states utilizing head motions, sound tunes, and color LED display panel. These expressive cues have been designed based on inspirations from dog-owner interactions [23]. In this study, both Sunflower robots used all the expressive modalities, however, the stationary Sunflower could not use gross body movements. Fig. 1 shows the Sunflowers within the robot house setting.

\section{$2.3 \quad$ Long-term study}

The results from this study were obtained as part of an ongoing long-term HRI study in the UH Robot house involving complex human-robot interaction scenarios. Throughout this study, a large amount of data was gathered and a full description and analysis of the general results from this study is currently being prepared, but for the purposes of this paper, a brief introduction follows:

The long-term study in the robot house aimed to convey the experience of longterm human robot interaction, by exposing participants to the robots in a series of episodic interactions. The user played the part of someone living in the robot house, with an Artificial Agent who inhabited different robot embodiments, one at a time. The agent's 'mind' could migrate between these different embodiments, see [24,25] for details. Each episode was framed a part of a specific day, with the user looking forward to performing some specific activities, within which the agent would assist by reminding the user of activities and previously inputted preferences; alerting the user to events in the environments like the doorbell going off, kettles and toasters being 
Sharing spaces, sharing lives - the impact of robot mobility on user perception of a home companion robot 5

finished; as well as function as a platform for communicating via Skype. In addition, the mobile robot was available for assisting in transporting objects.

Participants interacted with the agent in its robot embodiments in 9 sessions, two sessions a week, and filled in the questionnaire at the beginning of the $10^{\text {th }}$, debriefing session. Participants interacted with the stationary embodiment in all sessions. For the mobile embodiment, however, there were 2 sessions in which they did not interact with the mobile embodiment at all.

\subsection{Design and Experimental Control}

This study used a within-groups design, all participants interacted with both robot embodiments throughout the long-term study, using them for a variety of tasks. Both robots were capable of performing most socially assistive tasks, such as reminders and providing information but only the stationary robot could be used for communicating with another person via Skype, while the mobile robot could follow and guide the participant when walking around the robot house. Participants would have interactions through touch-screens with both robots for approximately the same amount of time

\subsection{Measures}

There were three means of data-capture. The first was the pictorial Inclusion of Other in the Self scale (IOS) [26]. This validated scale has been shown to correlate with feelings of closeness in human-human relationships and has also previously been used in HRI studies [27,28]. The second was a 5-point Differential Scale asking how close a participant felt two contrasting robot embodiments. These items were intended to explicitly make participant contrast the embodiments while considering their impressions of them.

Also used was the GODSPEED questionnaire[29], which was chosen as it is a robot specific scale, which addresses issues directly related to both evaluations of robotic embodiments as well as subjective impressions of robots. The final means of data capture was the use of open-ended questions in order to examine the specifics of participants' impressions of the robot. The relatively small sample-size made this a highly relevant approach.

\subsection{Hypotheses:}

H1: Participants will rate their relationship with the mobile robot as closer on the IOS scale compared to the stationary robot.

$\mathrm{H}_{0}$ : Participants will not distinguish between the two robot embodiments on the IOS Scale.

H2: Participants will report feeling close to the mobile robot on the Differential Scale item compared to the stationary Robot

$\mathrm{H} 2_{0}$ : Participants will not distinguish between the two robots on the Differential Scale item. 
6 Dag Sverre Syrdal, Kerstin Dautenhahn, Kheng Lee Koay, Michael L. Walters and Wan Ching Ho

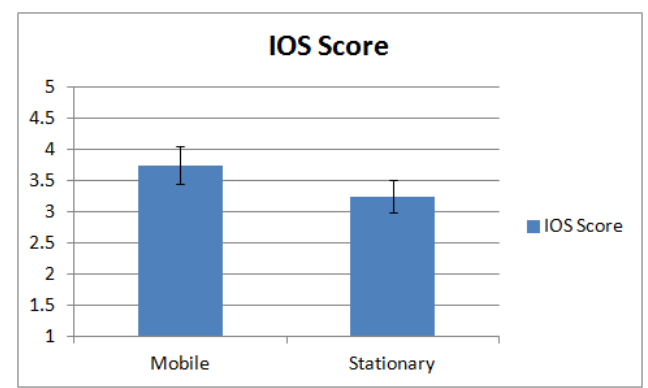

Fig. 2 IOS Scores

\section{Results}

\subsection{Inclusion of Self in the Other Scale}

Table 1. IOS Scores

\begin{tabular}{ccccc}
\hline Robot & Mean IOS (SE) & Mean difference & t-value (7 DF) & P \\
\hline Mobile & $3.75(.61)$ & & & \\
Stationary & $3.25(.56)$ & $.50(.33)$ & 1.27 & .17 \\
\hline
\end{tabular}

In the IOS scale, a score of 1 is indicative of no closeness, while a score of 6 is indicative of a high degree of closeness. While not significant for this number of participants, there was a trend in which participants rated their relationship with the mobile robot as closer than that with the stationary robot. This trend is shown in Fig. 2 and Table 1 , and had an effect size of $\mathrm{d}=.49$ observed power of .33 , with a critical $\mathrm{N}$ of 49 . One should also note that only 1 of the 8 participants rated the stationary robot closer than the mobile robot on the IOS Scale. While encouraging, however, this result did not allow us to fully reject null hypothesis 1 .

\subsection{Relative Closeness}

Participant responses to the Semantic Differential Item contrasting the two robot embodiments in terms of relative closeness was assessed using a one-sample t-test, testing for significant deviation from the middle value (3) of the contrast. A low score on this item was associated with feeling closer to the mobile robot and a high score with feeling closer to the stationary robot. The result is presented in Table 2 and suggests that the scores not only deviated significantly from the neutral value, but that 
Sharing spaces, sharing lives - the impact of robot mobility on user perception of a home companion robot

the direction of this deviation was caused by participants responding that they felt closer to the mobile robot, allowing us to reject null hypothesis 2.

Table 2. Relative Closeness

\begin{tabular}{lccc} 
& Mean Score (SE) & t-value & P \\
\hline Relative Closeness & $2.13(.30)$ & -2.97 & .02 \\
\hline
\end{tabular}

\subsection{Godspeed Questionnaire Results}

The results from the dimensions on the God speed Questionnaire can be found in Table 3 and Fig. 3, which shows a significant difference between the stationary and mobile robot along the Likeability dimension, suggesting that participants viewed the mobile robot as 'nicer' and more 'sympathetic' than the stationary robot. In addition, there was a trend in which participants tended to rate the mobile robot higher in terms of animacy. There were no differences between the robots in terms of anthropomorphism, intelligence and perceived safety. This allows us to reject null hypothesis 3 .

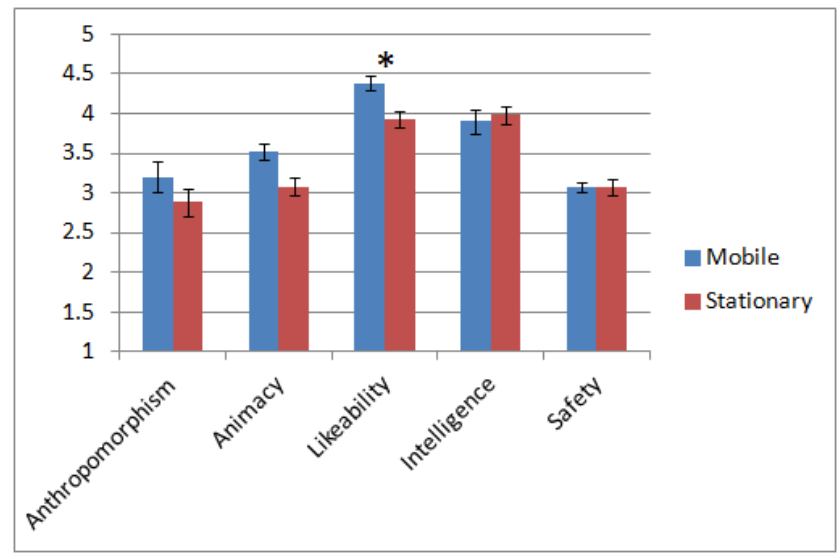

Fig. 3 Godspeed Questionnaire Scores

\section{Discussion}

\subsection{Summary of results}

The results are, overall encouraging, in terms of the research hypotheses. While not significant, there was a salient trend suggesting that participants rated their relationship with the mobile robot as closer than that with the stationary robot on the Inclusion of Self in Other scale. This trend, combined with the significant result for the Relative Closeness semantic differential item, as well as the significant difference between robots along the Likability scale on the Godspeed Questionnaire, suggests 
8 Dag Sverre Syrdal, Kerstin Dautenhahn, Kheng Lee Koay, Michael L. Walters and Wan Ching Ho

that interactions that involve moving in, and negotiating shared physical space, and the investment that understanding and learning about the robot's spatial behaviour involves, do play a role in the experienced building of a relationship between a domestic robot and a human user, even when comparing the results from two robots that have very similar interactional capabilities. It also seems that participants scored the two robots very closely in terms of intelligence and safety, suggesting that it was only in terms of relational measures that they viewed the mobile robot more favourably.

Table 3. Scores on the Godspeed Questionnaire

\begin{tabular}{cllccc}
\hline Dimension & \multicolumn{1}{c}{ Robot } & Mean(SE) & Mean Diff (SE) & t-value (7 DF) & p \\
\hline \multirow{2}{*}{ Anthropomorphism } & Mobile & $3.20(.38)$ & $.33(.53)$ & 0.61 & .56 \\
& Stationary & $2.88(.35)$ & & & \\
\multirow{2}{*}{ Animacy } & Mobile & $3.52(.21)$ & $.44(.31)$ & 1.41 & .20 \\
& Stationary & $3.08(.22)$ & & & \\
\multirow{2}{*}{ Likeability } & Mobile & $4.38(.18)$ & $.45(.19)$ & 2.35 & .05 \\
& Stationary & $3.93(.19)$ & & & \\
\multirow{2}{*}{ Intelligence } & Mobile & $3.90(32)$ & $.08(.21)$ & .36 & .73 \\
& Stationary & $3.98(.24)$ & & & \\
\multirow{2}{*}{ Safety } & Mobile & $3.08(.12)$ & .00 & .29 & 1 \\
\hline
\end{tabular}

This was echoed in terms of the open-ended comments that many participants offered, where they described the relationship between themselves and the stationary embodiment, as on in which they received and gave instructions to perform particular tasks, while the ability of the mobile embodiment to move and follow them as they were going about their business, conveyed a sense that they were sharing the experience or that they were collaborating to a larger extent, even if they conceded that there was no actual added practical benefit from this functionality.

\subsection{Implications and Future work}

The findings presented here support the notion that the gradual and rich interaction that can be had through negotiating shared physical space is conductive to feelings of closeness and general liking of the robot. This is in addition to the impact of embodiment as reported by Wainer et al. [19]. While there are risks and difficulties associated with the use of large-scale physical movement for robots, the advantages beyond that of the purely task-oriented should be considered.

The next step in this work is a thorough qualitative analysis of participants' interview responses to examine their conscious reasoning, and whether or not the ability of the robot to negotiate spaces were referenced in the debrief interviews that were held. While such an analysis is beyond the scope of this paper, early findings from this analysis are promising. In addition, further findings from the on-going analysis of this long-term study will also shed further light on the relationship between proxemic interactions and relationship building in Human-Robot Interaction. 
Sharing spaces, sharing lives - the impact of robot mobility on user perception of a home companion robot

\section{Acknowledgements.}

The research leading to these results has received funding from the European Union's Seventh Framework Programme (FP7/2007-2013) under grant agreement $n^{\circ}$ [287624], the ACCOMPANY project, and grant agreement $n^{\circ}$ [215554], the LIREC (LIving with Robots and intEractive Companions) project.

\section{References}

1. Dautenhahn K (2004) Robots We Like to Live With? - A Developmental Perspective on a Personalized, Life-Long Robot Companion. Proceedings of the 13th IEEE International Workshop on Robot and Human Interactive Communication(RO-MAN 2004):17-22

2. Cesta A, Cortellessa G, Giuliani MV, Pecora F, Scopelliti M, Tiberio L (2007) Psychological Implications of Domestic Assistive Technology for the Elderly. PsychNology Journal 5 (3):229-252

3. Montemerlo M, Pineau J, Roy N, Thrun S, Verma V (2002) Experience with a Mobile Robotic Guide for the Elderly. National Conference on Artificial Intelligence, AAAI, August, 2002

4. Huettenrauch H, Severinson Eklundh K (2002) Fetch-and-carry with CERO: Observations from a longterm user study with a service robot. Proceeding of the 11th IEEE International Workshop on Robot and Human Interactive Interactive Communication(RO-MAN 2001), Berlin, Germany Sept 25-27, 2002:158163

5. Danish Technological Institute (2012) James - Robot Buttler. http://robot.dti.dk/en/projects/james-robotbutler.aspx. Accessed 19 December 2012

6. Roy N, Baltus G, Fox D, Gemperle F, Goetz J, Hirsch T, Margaritis D, Montemerlo M, Pineau J, Schulte J Towards personal service robots for the elderly. In: Workshop on Interactive Robots and Entertainment (WIRE 2000), 2000. p 184

7. Fraichard T A short paper about motion safety. In: Robotics and Automation, 2007 IEEE International Conference on, 2007. IEEE, pp 1140-1145

8. Syrdal DS, Dautenhahn K, Woods SN, Walters ML, Koay KL 'Doing the right thing wrong' : Personality and tolerance to uncomfortable robot approaches. In: Procs of 15th IEEE Int Symp on Robot and Human Interactive Communication, RO-MAN 2006. pp 183-188

9. Syrdal DS, Dautenhahn K, Walters ML, Koay KL (2008) Sharing spaces with robots in a home scenario - anthropomorphic attributions and their effect on proxemic expectations and evaluations in a live HRI trial. In Proceedings of the 2008 AAAI Fall Symposium Symposium on AI in Eldercare: New Solutions to Old Problems

10. Heerink M, Kroese B, Evers V, Wielinga B (2006) The Influence of a Robot's Social Abilities on Acceptance by Elderly Users. Proceedings of The 15th IEEE International Symposium on Robot and Human Interactive Communication (RO-MAN06), Hatfield, UK, September 6-8, 2006:521-526

11. Kidd CD, Breazeal C Robots at home: Understanding long-term human-robot interaction. In: 2008 IEEE/RSJ International Conference on Intelligent Robots and Systems (IROS 2008), 22-26 Sept. 2008. pp 3230-3235. doi:10.1109/iros.2008.4651113

12. Huettenrauch H, Eklundh KS, Green A, Topp EA (2006) Investigating Spatial Relationships in HumanRobot Interaction. Proceedings of the IEEE/RSJ International Conference on Intelligent Robots and Systems(IROS 2006), Beijing, China:5052-5059 
10 Dag Sverre Syrdal, Kerstin Dautenhahn, Kheng Lee Koay, Michael L. Walters and Wan Ching Ho

13. Kendon A (1990) Conducting Interaction: Patterns of behavior in focused encounters, vol 7. Cambridge University Press, Cambridge, UK

14. Hall ET (1966) The Hidden Dimension. Doubleday, New York

15. Mehrabian A (1969) Significance of posture and position in the communication of attitude and status relationships. Psychological Bulletin 71 (5):359

16. Burgoon JK, Walther JB (1990) Nonverbal Expectancies and the Evaluative Consequences of Violations. Human Communication Research 17 (2):232-265

17. Stienstra J, Marti P Squeeze me: gently please. In: Proceedings of the 7th Nordic Conference on Human-Computer Interaction: Making Sense Through Design, 2012. ACM, pp 746-750

18. Cowley SJ, Macdorman KF (2006) What baboons, babies and Tetris players tell us about interaction: A biosocial view of norm-based social learning. Connection Science 18 (4):363-378

19. Wainer J, Feil-Seifer DJ, Shell DA, Mataric MJ Embodiment and human-robot interaction: A taskbased perspective. In: Robot and Human interactive Communication, 2007. RO-MAN 2007. The 16th IEEE International Symposium on, 2007. IEEE, pp 872-877

20. Kose-Bagci H, Ferrari E, Dautenhahn K, Syrdal DS, Nehaniv CL (2009) Effects of embodiment and gestures on social interaction in drumming games with a humanoid robot. Advanced Robotics 23 (14):1951-1996

21. Tapus A, Ţăpuş C, Matarić MJ (2008) User-robot personality matching and assistive robot behavior adaptation for post-stroke rehabilitation therapy. Intelligent Service Robotics 1 (2):169-183

22. Duque I, Dautenhahn K, Koay KL, Willcock I, Christianson B Knowledge-driven user activity recognition for a Smart House. Development and validation of a generic and low-cost, resource-efficient system. In: The Sixth International Conference on Advances in Computer-Human Interactions (ACHI 2013), Nice, France, February 24 - March 1 2013. p In press

23. Koay K, Lakatos G, Syrdal D, Gácsi M, Bereczky B, Dautenhahn K, Miklósi A, Walters ML Hey! There is someone at your door. A Hearing Robot using Visual Communication Signals of Hearing Dogs to Communicate Intent. In: IEEE ALIFE 2013 (The 2013 IEEE Symposium on Artificial Life), part of the IEEE Symposium Series on Computational Intelligence, Singapore, April 16-19 2013. IEEE,

24. Koay KL, Syrdal DS, Dautenhahn K, Arent K, Malek L, Kreczmer B (2011) Companion Migration Initial Participants' Feedback from a Video-Based Prototyping Study. In: Wang X (ed) Mixed Reality and Human-Robot Interaction, vol 47. Intelligent Systems, Control and Automation: Science and Engineering. Springer, pp 133-151

25. Koay KL, Syrdal DS, Walters ML, Dautenhahn K (2009) A User Study on Visualization of Agent Migration between Two Companion Robots. 13th International Conference on Human-Computer Interaction (HCII 2009). San Diego, CA, USA

26. Aron A, Aron EN, Smollan D (1992) Inclusion of Other in the Self Scale and the structure of interpersonal closeness. Journal of Personality and Social Psychology 63 (4):596

27. Mutlu B, Osman S, Forlizzi J, Hodgins J, Kiesler S Perceptions of ASIMO: an exploration on cooperation and competition with humans and humanoid robots. In: Proceedings of the 1st ACM SIGCHI/SIGART conference on Human-robot interaction, 2006. ACM, pp 351-352

28. Segura EM, Cramer H, Gomes PF, Nylander S, Paiva A Revive!: reactions to migration between different embodiments when playing with robotic pets. In: Proceedings of the 11th International Conference on Interaction Design and Children, 2012. ACM, pp 88-97

29. Bartneck C, Kulic D, Croft E, Zoghbi S (2008) Measurement Instruments for the Anthropomorphism, Animacy, Likeability, Perceived Intelligence, and Perceived Safety of Robots. International Journal of Social Robotics 1 (1):71-81 